\title{
Sobran cuchillos, falta el pescado. Auge del langostino patagónico y reactivación del conflicto social en Mar del Plata
}

\author{
Too many knives, too little fish. Patagonian shrimp boom and social conflict \\ resurgence in Mar del Plata
}

\author{
Diego Adrián Solimeno \\ diegosolimeno@hotmail.com \\ Universidad Nacional de Mar del Plata - Instituto \\ Superior de Formación Docente $\mathrm{N}^{\circ} 19$ - Grupo de \\ Estudios Regionales - GESMar, Argentina \\ Gonzalo Julián Yurkievich \\ Universidad Nacional de Buenos Aires - Universidad \\ Nacional de Mar del Plata - GESMar - Grupo de \\ estudios Regionales - Secretaría de Energía de la \\ Nación, Argentina
}

Recepción: 13 Junio 2020

Aprobación: 01 Septiembre 2020

Publicación: 02 Noviembre 2020

Cita sugerida: Solimeno, D. A. y Yurkievich, G. J. (2020). Sobran cuchillos, falta el pescado. Auge del langostino patagónico y reactivación del conflicto social en Mar del Plata. Geograficando, 16(2), e076. https://

doi.org/10.24215/2346898Xe076

\begin{abstract}
Resumen: En los últimos años, las poblaciones de langostino crecieron inusitadamente promoviendo que una buena parte de la flota fresquera y merlucera que tradicionalmente operaba en Mar del Plata se reorientara hacia esta especie de localización patagónica. El presente trabajo, apunta a demostrar que mientras las exportaciones pesqueras nacionales marcan récords históricos, la conflictividad social en la formación social y espacial pesquera de Mar del Plata se ha revitalizado motivada por la falta de pescado fresco descargado en tierra. En este sentido, analizamos: la evolución de las descargas de merluza y langostino en el país; el comportamiento de las distintas flotas y las características y recurrencia de la conflictividad social en Mar del Plata. A tales fines, trabajamos con bibliografía específica, estadísticas oficiales, prensa periódica, publicaciones especializadas del sector pesquero y entrevistas a informantes calificados.
\end{abstract}

Palabras clave: Pesca marítima, Conflicto social, Mar del Plata, Merluza, Langostino.

Abstract: In recent years, shrimp populations have grown unusually, encouraging a good part of the wet-fish and hake fleet traditionally operating in Mar del Plata to redirect their efforts towards this Patagonian species. This article aims to show that, while national fishery exports have set historical records, social conflict in the social and spatial fishing formation in Mar del Plata has been revitalized due to the lack of landed fresh fish. Thus, we study the evolution of hake and shrimp landings in the country, the behavior of different fleets, and social conflict features and recurrence in Mar del Plata. To this end, we have worked with specific bibliography, official statistics, periodical press, specialized publications from the fishing sector, and interviews with qualified informants.

Keywords: Maritime fishery, Social conflict, Mar del Plata, Hake, Shrimp. 


\section{A. INTRODUCCIón}

El final del siglo XX significó un momento de gran conflictividad para la actividad pesquera marítima argentina, principalmente asociada a la sobrepesca de la merluza hubbsi (principal especie capturada de nuestro mar) y a sus consecuencias para los trabajadores de plantas en tierra. El colapso de la especie se debió al aumento del esfuerzo pesquero, motivado por la masiva entrada de embarcaciones con mayor poder de captura y adelantos técnicos (principalmente europeas), y provocó graves conflictos ecológicos que terminaron generando prolongados procesos de conflictividad social. Estos conflictos tuvieron como principal escenario la formación social y espacial (FSE) ${ }^{1}$ pesquera de Mar del Plata, por ser esta terminal la que históricamente recibe los mayores volúmenes de descargas. La actividad había sufrido, para ese entonces, profundas mutaciones estructurales, que incluían, además del recambio de la flota mencionado, la precarización del trabajo y la concentración del empresariado pesquero (Allen, 2010; Mateo, Nieto y Colombo, 2011; Yurkievich, 2013). Estas transformaciones, implicaron que se considerase al recurso pesquero como moneda de cambio, o lo que Cóccaro, Le Bail, Gómez y Boetto (2000) catalogaron, inéditamente, como minería pesquera argentina. Los recursos públicos habían sido, así, trasnacionalizados y depredados en pos de intereses ajenos a los territorios locales.

La FSE marplatense rápidamente acusó los impactos del cambio de paradigma pesquero, y se transformó en un espacio de conflictos y contradicciones, en el que se hacían visibles las profundas dificultades relacionadas a la sustentabilidad económica, social, física y ecológica de la misma (Allen, 2010), con las consecuentes connotaciones políticas que esto implicaba.

La conflictividad social, vinculada a la actividad, encontró sus momentos más álgidos entre los años 1997 y 2007. En los años posteriores se dio una recuperación de los stocks de merluza hubbsi, a partir de la implementación de vedas y de la restricción a determinadas flotas por parte del Estado nacional (Yurkievich, 2013). Este hecho, sumado a una recuperación macroeconómica y a un nuevo envión exportador motivado por la devaluación del peso, condujo a una etapa de relativa calma, la cual se extendió hasta hace algunos años, cuando comenzaron a avizorarse nuevas aristas del conflicto social, asociadas a la falta de pescado fresco para procesar en las plantas de tierra de Mar del Plata.

A partir de esto, entendemos que la conflictividad actual en el sector no está relacionada con el estado de stock de la merluza hubbsi, como en décadas anteriores, sino que guarda relación con la disminución de los desembarques de pescado fresco en general, y de merluza hubbsi en particular. Este proceso, que se produce en un contexto de exportaciones récord para el sector, está impulsado por el traslado de parte de la flota fresquera hacia puertos patagónicos, en busca de otra pesquería más rentable, el langostino, de abundante stock biológico en los últimos años. La disminución de desembarques frescos en el puerto Mar del Plata se compensó con descargas provenientes de buques congeladores/factorías (que procesan el pescado abordo), los cuales generan menor cuantía de trabajo en tierra.

En este sentido, el presente trabajo busca demostrar que en los últimos años se ha reactivado el conflicto social en el puerto de Mar del Plata, a partir de la disminución de las descargas de pescado fresco, promovida por el traslado de parte de la flota de altura fresquera hacia los puertos patagónicos en busca del codiciado langostino austral.

\section{B. Metodología}

Para desarrollar el presente trabajo se utiliza una combinación de metodologías cuantitativas y cualitativas, entendiendo que es ésta la forma más adecuada para abordar los procesos sociales (Sautu, 2005). En este sentido, y en pos de lograr un análisis holístico de la problemática planteada, se propone 
adoptar lo que Knaf y Breitmayer (1989) denominan como triangulación metodológica, a partir de la combinación de metodologías cuantitativas y cualitativas.

De esta manera, observamos en primer término bibliografía específica para luego volcarnos al análisis de las estadísticas pesqueras generadas por la Secretaría de Agricultura, Ganadería y Pesca de la Nación en los últimos 30 años, lo cual nos permite estudiar la evolución de los desembarques objeto de estudio y sus implicancias en la ciudad de Mar del Plata. Por otra parte, relevamos noticias periodísticas de prensa periódica y medios especializados, a fines de observar los momentos de tensión y el discurso de los protagonistas. Finalmente, realizamos entrevistas a informantes clave, ya que entendemos, a partir de lo que plantea Daniel James (2004), que esta técnica "proporciona el acceso a informaciones empíricas básicas e imposibles de obtener en otras fuentes más tradicionales". En este sentido, agrega el autor:"el testimonio oral referido al ámbito mismo de la experiencia nos brinda valiosa información” (p. 125). Glassie (1982), por su parte, afirma que los testimonios orales nos conducen hacia una verdad más amplia que la atrapada en los fragmentos fácticos. De esta manera, a través de la combinación de técnicas y fuentes, pretendemos llegar a conclusiones rigurosamente fundadas sobre problemas novedosos y actuales, erigidos sobre antiguos problemas de carácter sistémico y estructural.

\section{Resultados Y Discusión}

\section{C.1. Cambios estructurales en la actividad y conflictos sociales a fines del siglo XX}

A partir de los años 80 comenzó una etapa de profundos cambios estructurales en la actividad pesquera argentina -que se potenciarían en la década siguiente-, que condujeron a la internacionalización del caladero, al desplazamiento de la pesca a la Patagonia, al recambio tecnológico, a la concentración empresarial y a la precarización del empleo, entre otros aspectos (Pradas, 2006). Si seguimos los lineamientos de este autor, a principios de los 90, y en el marco de las modificaciones a la ley de quiebras, algunas empresas líderes en el procesamiento se declararon en quiebra. De esta manera, alrededor de 6000 trabajadores fueron despedidos, y los grupos pesqueros sobrevivientes adquirieron los activos físicos que tuvieran algún valor (barcos y plantas) a un precio devaluado. Estos grupos empresariales adoptaron un modelo de integración vertical para organizar su producción. Los puertos patagónicos fueron su nueva base de operación,y complementaban al puerto de la ciudad de Mar del Plata. Un elemento fundamental de la nueva estrategia de acumulación estuvo dado por la masiva entrada de barcos congeladores-factoría al caladero argentino y por el aumento de la explotación de la mano de obra a través del sistema de cooperativas (Pradas, 2006).

La incorporación masiva de embarcaciones produjo un proceso de crecimiento acelerado de las capturas, que encontró su punto más álgido en las campañas de 1995 a 1997. El umbral de sostenibilidad del recurso fue superado durante varios años de la década de 1990 (Figura $N^{\circ} 1$ ).

FIGURA $\mathrm{N}^{\circ} 1$

Pesca marítima argentina, desembarques totales entre 1990 y $2019(\mathrm{t})$

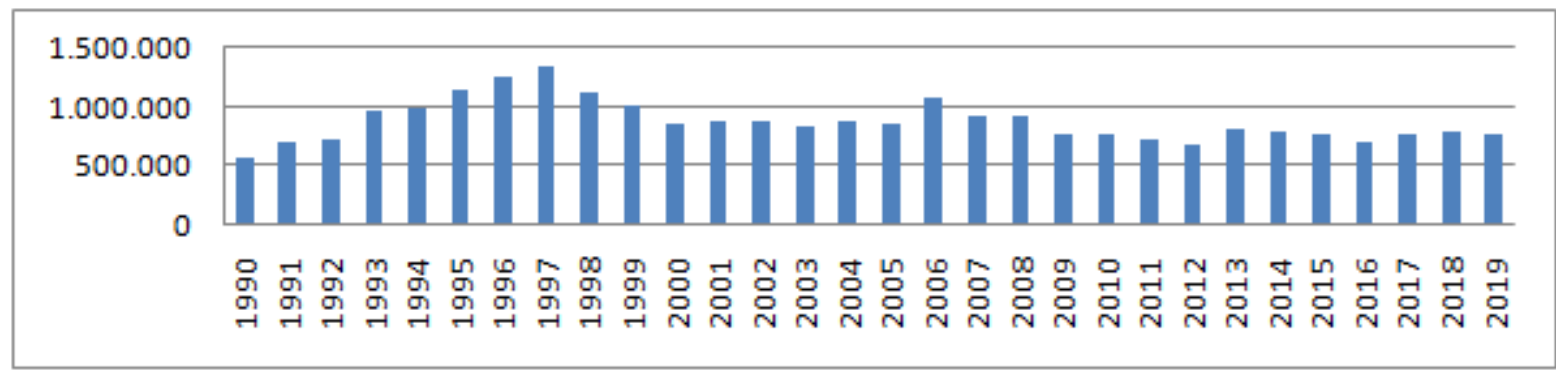

Fuente: Elaboración propia con base en datos de la SAGyP. 
En esta línea, Godelman (2004) argumenta que:

A principios de la década de 1990 la pesquería de merluza ya estaba en plena explotación, con un nivel de capturas de 390 mil toneladas (...) sin embargo, durante dicha década, por diferentes vías, pero principalmente por la llegada de 70 buques congeladores europeos, la capacidad de pesca de merluza se incrementó en un 150 por ciento y las capturas declaradas llegaron a 600 mil toneladas en 1996. Este descontrol, muchas veces acentuado por el otorgamiento indiscriminado y legalmente injustificable de permisos de pesca, llevó a la pesquería a una situación cercana al colapso (p. 2) (Figura N²).

Los stocks de merluza hubbsi entraron en crisis en el año 1997, y el gobierno debió establecer vedas y otros mecanismos de control para resguardar el caladero nacional, lo que provocó una reducción significativa en las capturas. Estas medidas pusieron en riesgo en forma directa a cerca de 20.000 trabajadores, y en forma indirecta a unas 200.000 personas en la ciudad de Mar del Plata. Comenzó así una profunda crisis política, económica y social, que llegó a su punto más crítico en las violentas jornadas de junio de 2000 (Nieto, 2005).

Entre los años 1999 y 2002 las vedas promovidas por la SAGPyA y la coyuntura macroeconómica desfavorable para la exportación generaron una reducción en la operatoria, que permitió una relativa recuperación del recurso. A partir de 2002, la nueva coyuntura favorable volvió a incrementar significativamente el esfuerzo pesquero en el marco de la nueva situación estructural de la rama, consolidada en el período anterior. Los conflictos sociales y laborales resurgieron con fuerza durante este nuevo período expansivo, principalmente durante la gran huelga de marineros de 2005 y durante el extendido ciclo de protesta y movilización de los trabajadores cooperativizados de 2007. En los años siguientes, si bien no se resolvieron los problemas de fondo en la actividad, no se registraron conflictos obreros reiterados y significativos, con la excepción del conflicto de marineros del año $2012{ }^{2}$

\section{C.2. Desembarques pesqueros en el siglo XXI}

La principal característica que muestran los desembarques pesqueros en este siglo es la estabilidadde las descargas en torno a las 800.000 t. Luego de atravesar años caóticos los desembarques marítimos se estabilizaron, sobre todo a partir del año 2008 (Figura $\mathrm{N}^{\circ} 1$ ). Este escenario respondió principalmente a la implementación, a partir de 2009, del sistema de Cuotas Individuales Transferibles de Captura (CITC) ${ }^{3}$ sobre la merluza hubbsi (principal especie capturada de nuestro mar).

FIGURA $\mathrm{N}^{\circ} 2$

Desembarques totales de merluza hubbsi entre 1990-2019(t).

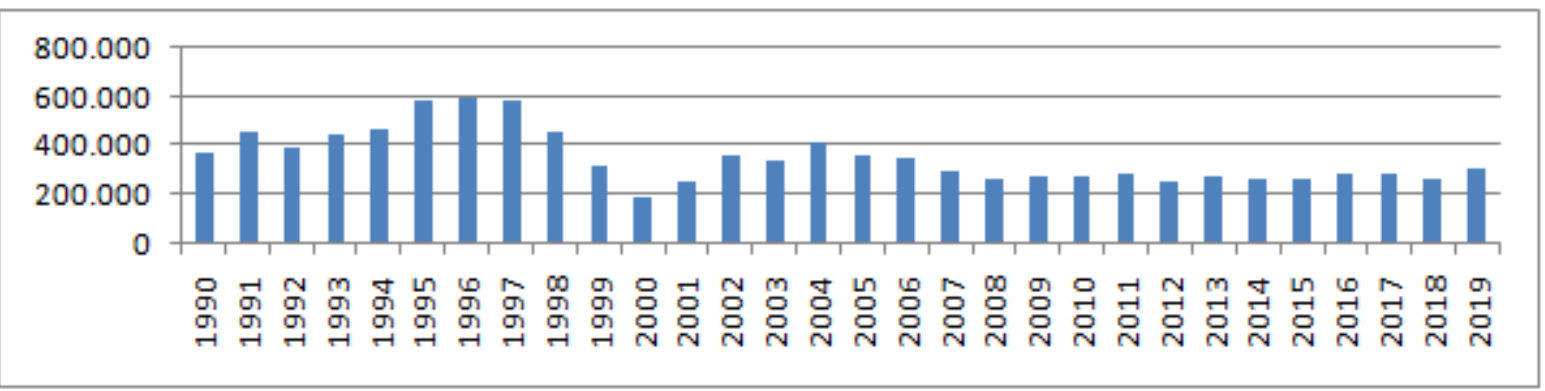

Fuente: Elaboración propia con base en datos de la SAGyP de la Nación.

La implementación de la cuotificación sobre la merluza provocó un reordenamiento de las capturas de la especie en torno a las $300.000 \mathrm{t}$ (Figura $\mathrm{N}^{\circ}$ 2), las cuales se repartieron entre los distintos puertos del país, y al mismo tiempo entre las distintas flotas, de modo que se recreó un escenario pesquero (en torno a esta especie) predecible y con pocas fluctuaciones. 


\section{C.3. Auge del langostino patagónico}

En el contexto anteriormente descripto, llama la atención, en los últimos diez años, el crecimiento de la pesquería de langostino, que provocó efectos sobre el resto de la actividad. Este crustáceo, de gran valor comercial, ${ }^{4}$ viene mostrando un crecimiento notorio en los volúmenes descargados desde el año 2008 hasta la actualidad. Las capturas de esta especie pasaron de menos de 50.000 t en 2008, a superar las 215.000 t para la campaña de 2019; máximo valor se registró en el año 2018, cuando alcanzaron las 250.000 t. Es decir, en diez años, las capturas prácticamente se quintuplicaron, lo que generó profundos cambios en la organización del sector pesquero nacional en general y marplatense en particular. Al mismo tiempo, este proceso posibilitó que los indicadores económicos de la actividad (sobre todo las exportaciones) crecieran, marcando récords históricos (Figura 3).

FIGURA No3

Exportaciones pesqueras, 2001-2019 (millones de dólares).

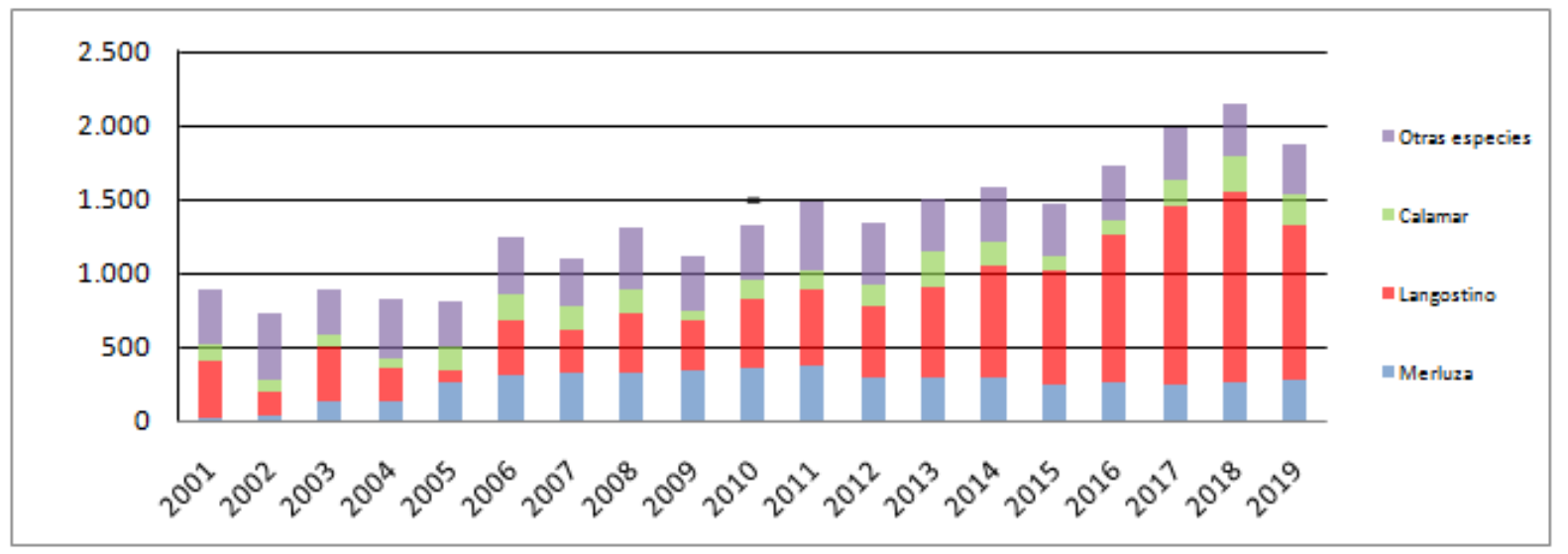

Fuente: Elaboración propia con base en datos de la SAGyP.

La pesca del langostino, con una temporada fuerte entre los meses de julio y febrero, viene generando desde el año 2008 cada vez más descargas, y, al mismo tiempo, llamando la atención de los principales actores de la actividad (frigoríficos, procesadoras, buques, marineros, etc.). Los buques que pueden volcarse a la pesca de esta especie, frente a márgenes de ganancias más elevados, reconvierten sus artes de pesca y migran hacia los puertos de la Patagonia, a fines de optimizar sus operaciones. De esta manera, obtienen un acceso más rápido a los caladeros y un regreso a tierra más acotado. La cercanía a puerto es fundamental para mantener la calidad de esta especie, la cual sufre un deterioro muy rápido. En este sentido, los puertos patagónicos presentan ventajas comparativas para concentrar el grueso de las descargas de este preciado crustáceo (Figuras 4 y 5 ). 
FIGURA 4

Distribución espacial de la pesquería de langostino en el Mar Argentino.

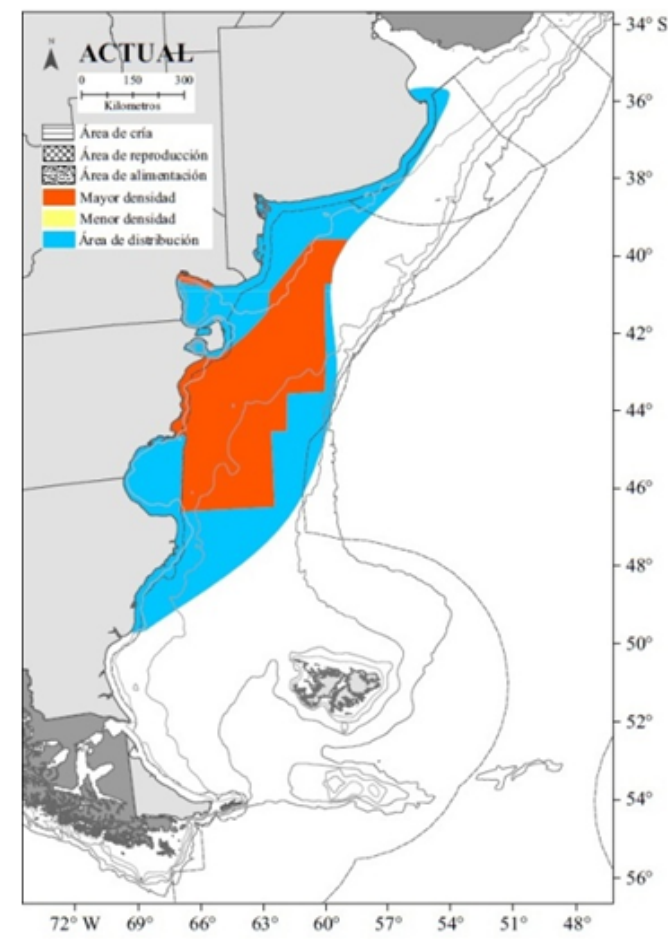

Fuente: "Estado del conocimiento biológico pesquero de los principales recursos vivos y su ambiente, con relación a la explotación hidrocarburífera en la Zona Económica Exclusiva Argentina y sus adyacencias.” (Allega, "et al.” 2020)

FIGURA 5

Principales puertos argentinos que reciben descargas de langostino, año 2019.

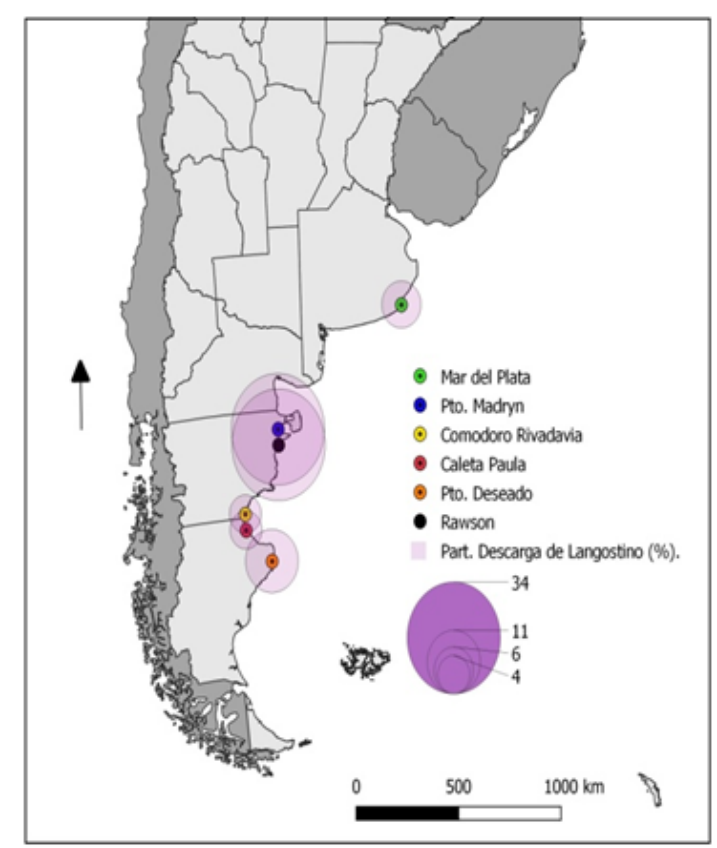

Fuente: Elaboración propia con base en datos de la SAGyP. 
En cuanto a la situación del puerto de Mar del Plata en relación a esta especie, por más que el mismo concentre el $46 \%$ de las capturas totales del país, ${ }^{5}$ las mismas están prácticamente abocadas a la merluza hubbsi, al calamar illex y al variado costero. La participación del langostino es muy baja en los desembarques, con volúmenes próximos al 6\% del total descargado en todo el país (Figura 6). Esta situación lo ubica en un lugar marginal respecto a otros puertos que históricamente tuvieron un rol secundario en la actividad pesquera nacional, como Rawson o Pto. Deseado. Sumado a esto, una de las flotas pesqueras más dinámicas de Mar del Plata (fresquera de altura) tiende, en los últimos años, a migrar hacia el sur para participar de la temporada langostinera, por lo que ha reducido sus descargas de merluza fresca en la ciudad.

FIGURA $N^{\circ} 6$

Desembarques de langostino por puerto año 2019.

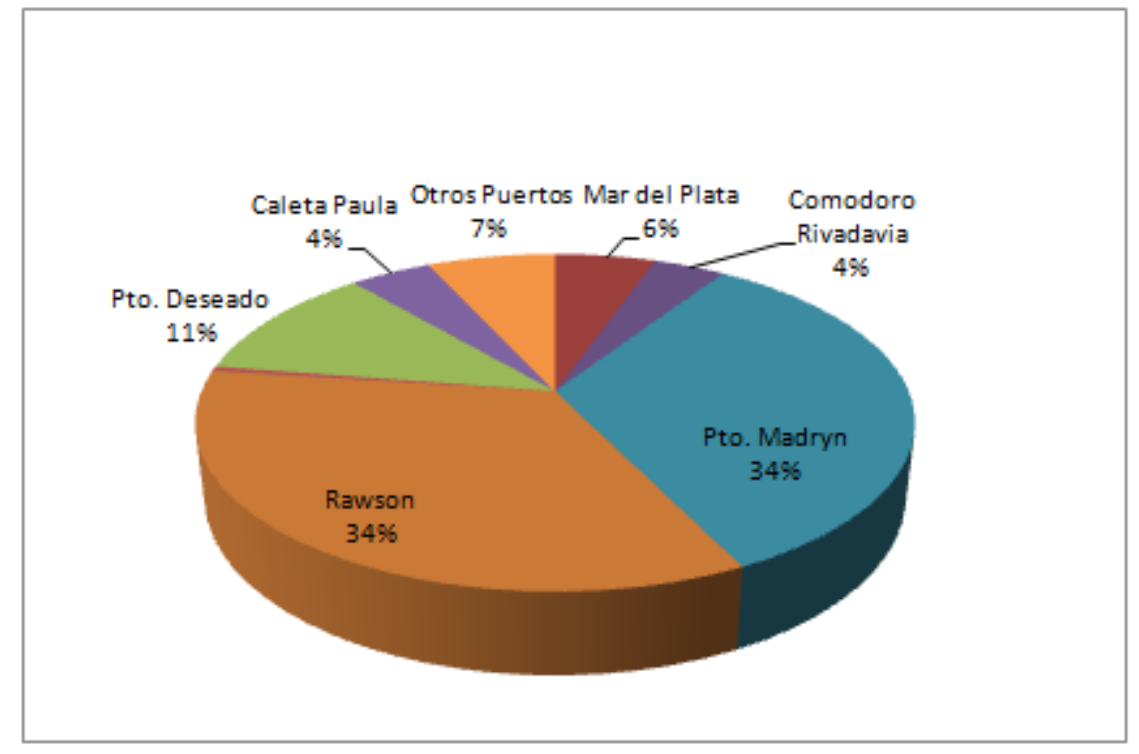

Fuente: Elaboración propia con base en datos de la SAGyP.

\section{C.4. Dinámica del puerto de Mar del Plata y éxodo de su flota fresquera}

Desde hace algunos años, el puerto de Mar del Plata recibe aproximadamente el 50\% de las capturas totales del mar argentino. Para el año 2019 ese porcentaje se ubicó en el 46\%. Al mismo tiempo, dos terceras partes de la meluza hubbsi capturada en nuestro mar (aproximadamente $200.000 \mathrm{t}$ ) se descargaron en este puerto, y de ellas, dos tercios en estado fresco. Al igual que las capturas totales de la especie, el porcentaje recibido por el puerto de la ciudad se viene comportando de manera constante desde hace más de una década (Figura 7).

Aunque este escenario parezca estable, cuando hurgamos en la composición de los desembarques de merluza hubbsi (flotas actuantes y estado de las capturas) en el puerto de Mar del Plata, vemos que las mismas han cambiado sensiblemente en los últimos años.

A partir del análisis de la Figura $\mathrm{N}^{\circ} 8$, podemos ver cómo la flota fresquera disminuyó sus desembarques en Puerto Mar del Plata desde el año 2008 en adelante, en estrecha vinculación con la aparición cada vez más significativa del langostino en las aguas nacionales. Los desembarques de esta flota cayeron de casi $250.000 \mathrm{t}$ en el año 2008 a cerca de 150.000 t en 2019. Esta disminución es muy significativa por los impactos que tiene en la cadena productiva local. La reducción de la participación de la flota no produjo una caída generalizada de los desembarques en el puerto de Mar del Plata, sino que los mismos se mantuvieron estables. Para sostenerlos (sobre todo los de merluza hubbsi), aumentaron marcadamente los desembarques de la flota congeladora/ factoría, los cuales pasaron de 60.000 t a casi 110.000 t en el mismo período. Es decir, que el puerto de Mar del Plata siguió concentrando porcentajes similares de descargas, pero las mismas arribaron ya procesadas, hecho que redujo la faena en las plantas locales. 
FIGURA $\mathrm{N}^{\circ} 7$

Desembarques de merluza hubbsi en Mar del Plata entre 2000 y $2019(\mathrm{t})$.

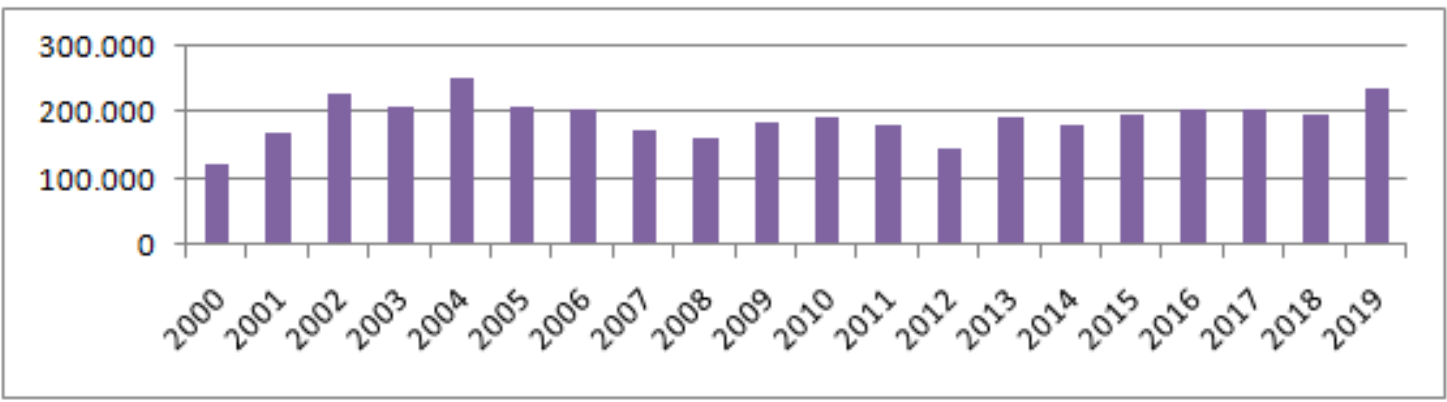

Fuente: Elaboración propia con base en datos de la SAGyP.

El auge que vienen teniendo las capturas de langostino impulsó a numerosas embarcaciones fresqueras, que operaban desde el puerto de Mar del Plata sobre la merluza hubbsi y otras especies, a solicitar permisos para emigrar hacia puertos patagónicos. La emigración de embarcaciones fresqueras fue haciéndose cada vez más intensa en la medida que la pesquería de langostino crecía, sobre todo en los últimos años.

FIGURA No8

Desembarques en Puerto Mar del Plata entre 2000 y 2019 ( $t$ ).

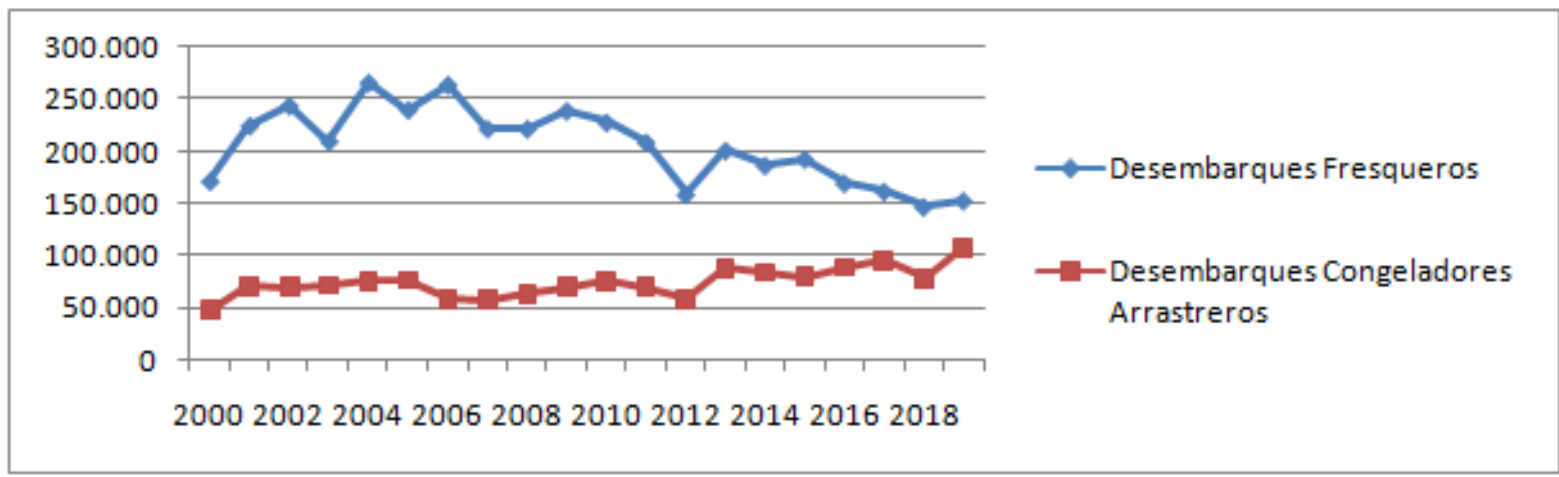

Fuente: Elaboración propia con base en datos de la SAGyP.

Los primeros síntomas de ese proceso nos llevan hasta el año 2014, cuando Carlos Mezzamico, del Sindicato Unido de Portuarios Argentinos (SUPA) y Rodolfo Chávez, del Sindicato Marítimo de Pescadores (SIMAPE), en una nota realizada por la Revista Puerto, si bien no anunciaban el número exacto de embarcaciones, ya pronosticaban: "todo aquel armador que puede escapar del puerto marplatense lo hace, aunque esa decisión repercuta en la merma de puestos de trabajo ligados a dicha flota, a bordo y en tierra”. (Garrone, R. 2014. párr.4)

En mayo de 2017, también la Revista Puerto daba cuenta de los preparativos que estaban realizando numerosos buques fresqueros para acoplarse a la temporada de langostino desde la Patagonia: "desde la Cámara de Armadores de Buques Fresqueros de Altura creen que serán unas 40 embarcaciones con permiso vigente para la pesca de langostino que participarán de la temporada”. (Garrone, 2017. párr. 4)

El sitio especializado Pescare también anunciaba la partida de ese número de embarcaciones, y al mismo tiempo afirmaba: "la preocupación asoma para sectores del trabajo en tierra como estibadores y obreros de plantas, que temen por la pérdida de volumen. Sin embargo, varios establecimientos locales tienen pactado procesar langostino que transportarán desde el sur". (Unos cuarenta barcos fresqueros emigran, 2017. párr. 4)

En el mes de mayo del año 2018, en entrevista del diario La Capital, Carlos Mezzamico (SUPA) manifestaba: 
estamos en vísperas de que se vayan al sur entre 60 y 70 barcos, y se les podrían sumar más buques, siempre y cuando logren los permisos ya pedidos. Esto es un problema gravísimo porque se quedaría mucha gente sin trabajo durante seis meses. (Advierten que entre 60 y 70 barcos, 2018, párr. 1)

Estos testimonios, sumados a lo que marcan las estadísticas, nos dan un panorama de la realidad que se vive hoy día en las inmediaciones del puerto de Mar del Plata. La emigración de la flota fresquera desequilibró las relaciones de producción que caracterizan a este espacio. Las descargas de merluza hubbsi y otras especies en fresco resultan fundamentales para el funcionamiento "armónico" del entramado de actividades que caracterizan a la actividad pesquera marplatense. Los distintos engranajes del sistema (descargas en puertos, transporte, procesado, embasado, insumos, etc.) se dinamizan en la medida en que ingresan al puerto descargas de pescado fresco.

En este sentido, y según surge del relato de Ángel, quien se desarrolla como capitán de un barco fresquero desde hace 10 años: "nosotros antes pescábamos merluza todo el año, pero desde 2015 también pescamos langostino para descargarlo en el puerto de Rawson. Nos trasladamos a principios de junio y si la temporada es buena regresamos recién a mediados de octubre". (Ángel, G. comunicación personal, 2 de septiembre de 2018)

Hernán, quien se desempeña como mecánico naval en la ciudad de Mar del Plata nos aclara:

los meses previos a la zafra de langostino se realizan muchas tareas de mantenimiento en las embarcaciones que se van a ir a langostino al sur, ya que por cinco o seis meses tienen que mantenerse operativos en el sur. Solamente frente a grandes averías vuelven a Mar del Plata. (Hernán, S. comunicación personal, 13 de marzo de 2020)

$\mathrm{Al}$ mismo tiempo nos aclara: "luego de estas reparaciones el trabajo en la ciudad disminuye mucho debido a la menor cantidad de embarcaciones operativas que quedan en el puerto”. (Hernán, S. comunicación personal, 13 de marzo de 2020)

\section{C.5. Reactivación del conflicto social por falta de pescado fresco}

No debe perderse de vista que lo que se viene describiendo forma parte de un mismo proceso, el cual en la última década ha provocado cambios en la actividad pesquera nacional. Es decir, la aparición de langostino en abundancia provoca gran interés en el sector, el cual se vuelca a su búsqueda. Un número significativo de buques fresqueros marplatenses migra hacia puertos patagónicos para poder dedicarse de manera temporal a esta faena. Los desembarques de merluza fresca en puerto Mar del Plata pasan a ser cubiertos por la flota factoría/congeladora. Este cambio en las descargas provoca la interrupción de las cadenas productivas tradicionales en este puerto. Como consecuencia, se reactiva el conflicto social, ya no por falta de stock de merluza (como a fines de los años 90 y principios de los 2000), sino por un cambio en las condiciones de producción de la rama. Esta situación provocaque en los últimos años se observe una reactivación de los conflictos gremiales en el sector pesquero de la ciudad de Mar del Plata, asociada a estos procesos.

Uno de los episodios más críticos ocurrió durante el mes de mayo de 2018, cuando distintos gremios bloquearon las terminales 2 y 3 del puerto de Mar del Plata con el objetivo de visibilizar los reclamos por la pérdida de puestos de trabajo en la rama del filet. En la revista digital Infocielo, Juan Vargas, de Camioneros, advertía:

muchas personas van a perder el trabajo si los barcos emigran a la Patagonia por la pesca del langostino (...) ya hubo cincuenta despidos y las pérdidas de los puestos de trabajo se intensificarían en los próximos meses (...) queremos sentarnos en una mesa de negociaciones porque los trabajadores la están pasando muy mal (...) el puerto mueve el $80 \%$ del mercado interno de la ciudad y eso no lo ven; nos ven como una ciudad turística pero hay mucha gente que depende del puerto.(Trabajadores bloquean el puerto de Mar del Plata, 2018. párr. 1) 
La nota proseguía afirmando que: “...el conflicto proviene porque una parte de la flota pesquera marplatense piensa retirarse a la Patagonia para dedicarse a la pesca del langostino". (Trabajadores bloquean el puerto de Mar del Plata, 2018. párr. 1)

El mismo dirigente afirmaba al diario La Capital: "el bloqueo al puerto de Mar del Plata es para exigir la conformación de una mesa de diálogo que evite que los barcos se vayan al sur a pescar langostino y dejen sin trabajo a las plantas locales" (Baradel participó del bloqueo del puerto, 2018. párr.3)

En este sentido Edith, quien se desempeña como filetera en cooperativas de trabajo desde hace 10 años afirma: "hace 4 o 5 años que llega cada vez menos merluza para cortar a las plantas. Pase de trabajar 5 o 6 días por semana a solamente 2 o 3". Refuerza argumentando: "los meses que los barcos se mudan al sur se hace difícil conseguir pescado para cortar". (Edith, A., comunicación personal, 15 de abril de 2020)

El 3 de diciembre de 2018, el diario La Capital remarcaba:

se agudiza la crisis en el Puerto y los gremios temen una ola de despidos (...) dirigentes sindicales de los principales gremios advierten que la situación "cada año está peor". Un invierno marcado por la ausencia de 70 barcos, la imposibilidad de acceder a la pesca de nuevas especies y los altos índices de inflación configuran un escenario que temen que termine con un profundo conflicto social (...) si los barcos se van al sur habrá alrededor de 8.000 trabajadores perjudicados en Mar del Plata, especialmente estibadores, transportistas y fileteros. (Se agudiza la crisis en el Puerto, 2018. párr.1)

En este contexto, surgieron conflictos puntuales en diversas plantas que debieron reducir su personal por falta de pescado fresco.

Durante marzo de 2019 estalló un conflicto en la firma Sebastián Gaboto y El Marisco, ambas del empresario Antonio Di Leva. Con respecto al mismo, Roberto Garrone (2019) para Revista puerto afirmaba:

este martes un grupo de trabajadores de la pesquera Sebastián Gaboto y frigorífico El Marisco, ambas de Antonio Di Leva, enrolados bajo el Anexo Pyme, volvieron a manifestarse en la vía pública con quema de cubiertas ante la imposibilidad de firmar el aumento del salario garantizado. Ese era el compromiso asumido por la empresa el jueves por la tarde en la sede de la Comisaría Tercera, junto con delegados y dirigentes del SOIP (Sindicato Obrero de la Industria del Pescado), luego que los trabajadores se manifestaran frente a El Marisco y denunciaran la crisis laboral que atraviesan, sin posibilidades de superar el garantizado a partir de la escasa y discontinua convocatoria a trabajar.(párr. 1)

Carlos Mata, presidente de la empresa relataba en la misma nota:

hemos traído materia prima desde el sur y pensamos seguir haciéndolo para dar trabajo en Mar del Plata, pero el sector de corte con el fenómeno del langostino estaba con una garantía (...) no nos gusta esta situación porque no queremos cerrar. Vamos a mantener a más de cuarenta trabajadores entre peones, envasadoras y camaristas pero necesitamos reestructurar el trabajo del corte porque teníamos muy poca actividad. (Garrone, R. op. Cit. párr. 5.)

Revista Puerto en un artículo de febrero de 2018 también anunciaba que uno de los principales empresarios de la actividad, Alberto Valastro, había reducido a una cuarta parte su nómina de trabajadores a partir de los problemas de rentabilidad que presentaba la actividad fresquera vinculada a la merluza hubbsi. La estrategia del empresario, no solo pasó por la reducción de su personal en tierra, sino directamente por desprenderse de la mayor parte de sus buques fresqueros. Este medio especializado afirmaba:

el cambio de modelo, la supremacía del esquema congelador por sobre el fresquero, parece no tener marcha atrás y comienzan a verse las consecuencias en la industria pesquera marplatense (...). En la pesca marplatense ya ni siquiera se pueden retener los puestos que quedan. Nada resiste el aluvión que expulsa trabajadores a la calle. (Garrone, R. 2018 a, párr.17)

Una nota muy representativa de la realidad del sector, asociada a la hipótesis de trabajo de este artículo, es la publicada por Roberto Garrone (2018 b) en la Revista Puerto:

la estadística general que monitorea los desembarques de merluza marca un descenso en el primer semestre que supera el $15 \%$, por una reducción de los aportes que la flota fresquera realiza a partir de capturas sobre el efectivo norte y la zona común de pesca (...) Cafrexport reúne a 16 empresas y el balance generalizado del mes de junio arrojó una reducción en el nivel de actividad que alcanzó el $60 \%$ en algunos casos y en otros no bajó del $40 \%$ (...) en dos semanas los referentes sindicales 
volverán a reunirse con el Ministerio de Trabajo como mediador entre los armadores y los sindicatos para intentar que el puerto de Mar del Plata recobre la operatividad que, como la merluza, brilla por su ausencia. (párr. 1)

Más allá de los conflictos sindicales (vinculados al sector industrial), también viene siendo cada vez más frecuente que comerciantes de la zona del puerto manifiesten su preocupación respecto a la caída de la actividad en la zona. Históricamente la calle 12 de Octubre (principal calle comercial de la zona portuaria) muestra en su volumen de actividad un reflejo del vigor de la actividad pesquera marplatense. En este sentido, el portal digital de noticias 0223 entrevistó a comerciantes que afirmaban:

en los últimos 6 meses cerraron alrededor de 15 locales, la relación entre esta realidad y la caída de la actividad pesquera es total, los barcos están en el sur, el trabajo diario del pescado está muy tranquilo. Estamos viendo cómo hacer para aguantar este invierno así como estamos. (12 de Octubre, una calle que se apaga, 2019. párr. 1)

\section{Conclusiones}

La actividad pesquera nacional atraviesa un periodo de auge en términos macroeconómicos, sobre todo, por el valor total de sus exportaciones, próximo a los 2 mil millones de dólares en el año 2019. Estos valores se obtienen, principalmente, a partir del crecimiento que muestra la pesquería de langostino desde hace diez años.

Esta situación se contrasta con la reaparición de conflictos obreros, que se manifiestan en la FSE pesquera de Mar del Plata. Como pudimos observar, los mismos se generan, sobre todo, a partir de la falta de dinamismo que viene mostrando la factura en tierra en esta ciudad, lo que también afecta indirectamente a la actividad comercial y de servicios en la zona portuaria. Estas problemáticas (que aun pueden profundizarse) tienen origen, entre otras cosas, en las relaciones sociales de producción que caracterizan a la actividad pesquera en este espacio geográfico en particular.

Como se mostró en el desarrollo del trabajo, para que la actividad se muestre dinámica y sin conflictos sociales latentes o manifiestos es preciso que los volúmenes de pescado fresco que llegan a la ciudad de Mar del Plata (sobre todo los de merluza hubbsi) se sostengan en niveles que permitan dinamizar los mercados de trabajo. Las mermas en estas descargas han producido, décadas atrás, intensa conflictividad y severas crisis en el sector. Los conflictos que marcaron el final de la década del 90 y los principios del siglo XXI tuvieron estas características, asociadas a la falta de stock de merluza hubbsi producto de la sobrepesca de la especie.

La recuperación de stocks biológicos y la estabilización de los desembarques, a partir de la implementación de las CITC (Cuotas Individuales Transferibles de Captura), promovieron una reducción de la conflictividad social en el puerto de Mar del Plata.

La irrupción del langostino y el movimiento de la flota fresquera hacia el sur pusieron en jaque nuevamente a los actores más vulnerables de la cadena pesquera local, debido a una nueva reducción de las descargas de pescado fresco. Ante esta situación comenzaron a ser más frecuente las manifestaciones de obreros del sector, los cuales intervinieron en el territorio, muchas veces a través de la acción directa, para evidenciar sus reclamos. Los mismos, en la medida que se hicieron reiterados, comenzaron a ser relevados sistemáticamente por la prensa periódica local y especializada.

El desarrollo de este trabajo nos ha permitido demostrar cómo a pesar de la estabilización de la actividad (entre otras cosas por la introducción de la cuotificación) y la existencia de stock de merluza disponible, el conflicto social sigue latente en el puerto de la ciudad de Mar del Plata. En este sentido, el mismo está más asociado a las relaciones sociales de producción que se establecieron en la actividad, que a la disponibilidad biológica de una especie. La profundización de este proceso pondría en jaque, nuevamente, la sustentabilidad económica, social y física de la FSE pesquera marplatense. A diferencia de décadas anteriores, la misma no se produciría por problemas vinculados a la sustentabilidad ecológica de la actividad (sobrepesca de merluza), sino por la merma de la operatoria de la flota fresquera abocada a la pesca de langostino. 
Por otro lado, nos gustaría reflexionar acerca del crecimiento de la pesquería de langostino y del aumento desmedido del esfuerzo pesquero sobre esta, a cuya búsqueda se siguen incorporando buques cada año. Repasar la historia de la pesca marítima en nuestro país nos puede ayudar a no repetir errores en torno al manejo de una especie y su agotamiento, lo cual termina generando impactos sociales y espaciales en las ciudades portuarias. La importancia de esta especie en la economía pesquera, amerita planes de manejo que contemplen no solo la sustentabilidad ecológica de la misma, sino el bienestar de todos los actores sociales involucrados en la actividad, sobre todo, sus eslabones más débiles, los asalariados de mar y de tierra. Al mismo tiempo, habría que replantear el rumbo que implica la evolución de este tipo de pesquerías. El desarrollo de la pesca del langostino involucra la captura y exportación, prácticamente sin valor agregado ni eslabonamientos productivos en los puertos de descargas. De esta manera, pareciera que se estarían replicando nuevamente escenarios de "minería pesquera", los cuales impactan en los guarismos macroeconómicos, pero no velan por la integridad de todos los actores sociales involucrados.

\section{REFERENCIAS}

12 de Octubre, una calle que se apaga. (26 de mayo de 2019). Portal 0223. Recuperado de: https://www.0223.com.ar/nota/2019-5-26-14-12-0-12-de-octubre-una-calle-que-se-apaga-el-ritmo-de-la-c risis-pesquera-en-6-meses-cerraron-15-comercios

Advierten que entre 60 y 70 barcos se irán a pescar al sur durante el invierno. (4 de mayo de 2018). Diario La Capital. Recuperado de: https://www.lacapitalmdp.com/advierten-que-entre-60-y-70-barcos-se-iran-a-pescar-al-sur-du rante-el-invierno/

Allega, L.; Braverman, M.; Cabreira, A.G.; Campodónico, S.; Carozza, C.R.; Cepeda, G.D.; Colonello, J.H.; Derisio, C.; Di Mauro, R.; Firpo, C.A.; Gaitán, E.N.; Hozbor, M.C.; Irusta, C.G; Ivanovic, M.; Lagos, N.; Lutz, V.A.; Marí, N.R.; Militelli, M.I.; Moriondo Danovaro, P.I.; Navarro, G.; Orlando, P.; Pájaro, M.; Prandoni, N.; Prosdocimi, L.; Reta, R.; Rico, R.; Riestra, C.M.; Ruarte, C.; Schejter, L.; Schiariti, A.; Segura, V.; Souto, V.S.; Temperoni, B.; Verón, E. (2020). Estado del conocimiento biológico pesquero de los principales recursos vivos y su ambiente; con relación a la exploración hidrocarburifera en la Zona Económica Exclusiva Argentina y adyacencias. Mar del Plata: Instituto Nacional de Investigación y Desarrollo Pesquero INIDEP.

Allen, A. (2010). ¿Sustentabilidad ambiental o sustentabilidad diferencial? La reestructuración neoliberal de la industria pesquera en Mar del Plata, Argentina. Revista de Estudios Maritimos y Sociales, año 3, No3, GESMAR; Mar del Plata.

Baradel participo del bloqueo del puerto. (16 de mayo de 2018). Diario La Capital. Recuperado de: https://www.lacap italmdp.com/baradel-participa-del-bloqueo-del-puerto/

Cóccaro, J. M., Le Bail, J., Gómez, O. y Boetto, A. (2000). “La minería pesquera ¿argentina?”. En Actas del Segundo Encuentro Internacional Humboldt. Mar del Plata.

Garrone, R. (20 de agosto de 2014). Marineros y estibadores advierten un desplome en la actividad fresquera marplatense. Revista Puerto. Recuperado de: https://revistapuerto.com.ar/2014/08/marineros-y-estibadoresadvierten-un-desplome-en-la-actividad-fresquera-marplatense/

Garrone, R. (18 de mayo de 2017). Desde Mar del Plata, éxodo de fresqueros al langostino. Revista Puerto. Recuperado de: https://revistapuerto.com.ar/2017/05/desde-mar-del-plata-exodo-de-fresqueros-al-langostino/

Garrone, R. (14 de febrero de 2018 a). Valastro se desliga del fresco y lo sufren los trabajadores. Revista Puerto. Recuperado de: https://revistapuerto.com.ar/2018/02/valastro-se-desliga-del-fresco-y-lo-sufren-los-trabajador es/

Garrone, R. (20 de julio de 2018 b). La falta de merluza redujo la actividad de frigoríficos sin barcos propios. Revista Puerto. Recuperado de: https://revistapuerto.com.ar/2018/07/la-falta-de-merluza-redujo-actividad-de-frigorif icos-sin-barcos-propios/ 
Garrone, R. (27 de marzo de 2019). En Mar del Plata se extiende el conflicto en pesqueras de Antonio Di Leva. Revista Puerto. Recuperado de: https://revistapuerto.com.ar/2019/03/en-mar-del-plata-se-extiende-el-conflicto-en-pe squeras-de-antonio-di-leva/

Glassie, H. (1982). Passing the time in Ballymenone: Culture and history of anulster community. Pennsylvania: University of Pennsylvania press.

Godelman, E. (2004). Propuesta de ordenamiento de la pesquería de merluza en Argentina. CeDePesca, Argentina.

James, D. (2004). Doña María. Historia de vida memoria e identidad politica. Buenos Aires: Manantial.

Knaf, A. y Breitmayer, B. (1989). "Triangulation in cualitative research" en Morse J. Qualitative nursing research: a contemporary dialogue. Aspen: Aspen ed.

Mateo J. (2003).Deespaldas al mar: la pesca en el Atlántico sur (siglos XIX XXX) (Tesis doctoral). Barcelona: Universitat Pompeu Fabra, (mimeo).

Mateo, J. A., Nieto, A. y Colombo, G. (2011). Precarización y fraude laboral en la industria pesquera marplatense. El caso de las «cooperativas» de fileteado de pescado. Estado actual de la situación y evolución histórica de la rama, 1989-2010. En Las condiciones de trabajo en la provincia de Buenos Aires (Vol. 2, pp. 177-302). La Plata: Ministerio de Trabajo de la provincia de Buenos Aires.

Nieto, A. (2005). Lucha de calles en el puerto de Mar del Plata. 28 y 29 de junio de 2000. En Actas X Jornadas Interescuelas Departamentos de Historia. UNR-Rosario.

Nieto, A. y Colombo, G. (2009). Lucha de calles en la industria de la pesca Una interpretación del porqué de su regularidad (1997-2007). Conflicto Social, 2 (1), 168-194. Recuperado de https://publicaciones.sociales.uba.a r/index.php/CS/article/view/446.

Pradas, E. (2006). Un acercamiento a la problemática pesquera marplatense. Mar del Plata: El Mensajero.

Santos, M. (1996). De la totalidad al lugar. Barcelona: Oikos-Tau Ediciones.

Sautu, R. (2005). Todo es teoría. Buenos Aires: Lumiere.

Se agudiza la crisis en el Puerto y los gremios temen una ola de despidos. (3 de diciembre de 2018). Diario La Capital. Recuperado de: https://www.lacapitalmdp.com/se-agudiza-la-crisis-en-el-puerto-y-los-gremios-temen-una-olade-despidos/

Trabajadores bloquean el puerto de Mar del Plata para frenar los despidos. (16 de mayo de 2018). Portal Infocielo. Recuperado de: https://infocielo.com/langostinos/trabajadores-bloquean-el-puerto-mar-del-plata-frenar-los-d espidos-n91582

Unos cuarenta barcos fresqueros emigran de Mar del Plata para pescar langostino. (22 de mayo de 2017). Portal Pescare. Recuperado de: https://www.pescare.com.ar/unos-cuarenta-barcos-emigran-de-mar-del-plata-para-pescar-lang ostino/

Yurkievich G. (2008). Crónica de un conflicto anunciado. Otro capítulo de la lucha por la registración laboral en el Puerto de la ciudad de Mar del Plata. Revista de Estudios Marítimos y Sociales. Número I, GESMAR, Mar del Plata.

Yurkievich, G. (2007). Configuración social y espacial de la actividad pesquera maritima en el barrio Puerto de la ciudad de Mar del Plata. Auge, declinación y decadencia. En Actas III Jornadas de Geografía Económica, Facultad de Humanidades, Universidad Nacional de Mar del Plata.

Yurkievich, G. (2010). Transformación estructural, conflictividad social y deterioro espacio-ambiental en el Puerto de la ciudad de Mar del Plata. 1997-2007. Revista de Estudios Maritimos y Sociales, año 3, No3, GESMAR; Mar del Plata.

Yurkievich, G. (2013). Pesca y puerto en la ciudad de Mar del Plata. Relaciones íntimas entre una actividad económica transformada y un espacio deteriorado. Estudio Socioterritoriales, (14), 37-68.

Yurkievich, G. y Nieto, A. (2014). Organización y lucha obrera en la Argentina contemporánea: La experiencia del Sindicato Marítimo de Pescadores (2000 - 2012). Trabajo y Sociedad, (23), 179-204.

Ministerio de Agricultura, Ganadería y Pesca. Subsecretaria de Pesca y Acuicultura 


\section{Notas}

1 La categoría de formación social y espacial pesquera marplatense fue utilizada por Yurkievich (2010) a partir de la conceptualización realizada por Santos (1996) en "De la totalidad al lugar". El autor considera que los modos de producción se tornan concretos en una base territorial, a través de las formas espaciales. A partir de allí, establece que las relaciones entre espacio y formación social se forjan en espacios particulares. Es por esto que se propone reunir las categorías de formación económico-social y formación espacial por una más totalizadora y amplia como la de formación social y espacial.

2 Para ampliar sobre la conflictividad obrera en el sector véase Nieto y Colombo (2009), Yurkievich y Nieto (2014) y Yurkievich (2008).

3 A partir de la Implementación de la Ley Federal de Pesca №24.922/98 y del Régimen Federal Pesquero ley 26.386. Las cuotas individuales transferibles son una concesión temporal que otorga el Estado, a través del Consejo Federal Pesquero, a favor del titular de un permiso de pesca, que habilita a su dueño a la captura de un porcentaje de la captura máxima permisible de una especie (principalmente las diferentes especies de merluza -hubbsi, polaca, negra, de cola, etc.).

4 Según datos de la SAGyP (Informe de Exportaciones e importaciones pesqueras, 2018) de la Nación para el año 2018, el precio promedio de la tonelada de langostino fue de U\$S 7.013, mientras que el valor de la merluza hubbsi fue de U \$U 2.684 .

5 Según las estadísticas de la SAGyP de la Nación, en el año 2019 los desembarques en el puerto de Mar del Plata alcanzaron un $46 \%$. 